\title{
Metabolic Pathway Alterations that Support Cell Proliferation
}

\author{
M.G. VANDER Heiden ${ }^{1,2}$, S.Y. Lunt ${ }^{1}$, T.L. DaYton ${ }^{1}$, B.P. Fiske ${ }^{1}$, W.J. IsRaelsen ${ }^{1}$, \\ K.R. Mattaini ${ }^{1}$, N.I. Vokes $^{1}$, G. Stephanopoulos ${ }^{3}$, L.C. Cantley ${ }^{4}$, \\ C.M. Metallo ${ }^{3,5}$, and J.W. Locasale ${ }^{4}$ \\ ${ }^{1}$ Massachusetts Institute of Technology, Koch Institute for Cancer Research and Department of Biology, \\ Cambridge, Massachusetts 02139 \\ ${ }^{2}$ Dana-Farber Cancer Institute, Medical Oncology, Boston, Massachusetts 02115 \\ ${ }^{3}$ Massachusetts Institute of Technology, Department of Chemical Engineering, Cambridge, \\ Massachusetts 02139 \\ ${ }^{4}$ Harvard Medical School, Department of Systems Biology, Boston, Massachusetts 02115 \\ ${ }^{5}$ University of California-San Diego, Department of Bioengineering, La Jolla, California 92093 \\ Correspondence: mvh@mit.edu
}

\begin{abstract}
Proliferating cells adapt metabolism to support the conversion of available nutrients into biomass. How cell metabolism is regulated to balance the production of ATP, metabolite building blocks, and reducing equivalents remains uncertain. Proliferative metabolism often involves an increased rate of glycolysis. A key regulated step in glycolysis is catalyzed by pyruvate kinase to convert phosphoenolpyruvate (PEP) to pyruvate. Surprisingly, there is strong selection for expression of the less active M2 isoform of pyruvate kinase (PKM2) in tumors and other proliferative tissues. Cell growth signals further decrease PKM2 activity, and cells with less active PKM2 use another pathway with separate regulatory properties to convert PEP to pyruvate. One consequence of using this alternative pathway is an accumulation of 3-phosphoglycerate (3PG) that leads to the diversion of 3PG into the serine biosynthesis pathway. In fact, in some cancers a substantial portion of the total glucose flux is directed toward serine synthesis, and genetic evidence suggests that glucose flux into this pathway can promote cell transformation. Environmental conditions can also influence the pathways that cells use to generate biomass with the source of carbon for lipid synthesis changing based on oxygen availability. Together, these findings argue that distinct metabolic phenotypes exist among proliferating cells, and both genetic and environmental factors influence how metabolism is regulated to support cell growth.
\end{abstract}

All cells rely on a source of nutrients for growth and survival. These nutrients are taken up from the environment and directed into metabolic pathways to maintain homeostasis and fuel cell-type-specific functions. For all cells, these processes include maintenance of ion gradients across membranes, transport of materials between intracellular compartments, and other housekeeping functions such as protein turnover. Many of these processes are thermodynamically unfavorable and thus are coupled to ATP hydrolysis as a source of free energy. To provide metabolic support for these functions, cells have evolved pathways, such as the oxidative metabolism of glucose, that when coupled to mitochondrial oxidative phosphorylation can efficiently generate ATP from available nutrients.

Proliferating cells, including cancer cells, metabolize nutrients to support these same housekeeping functions. They also must take up additional nutrients, metabolize these nutrients through pathways that produce ATP, and generate all the components necessary to duplicate the mass of the cell and allow for cell division (Fig. 1). For many unicellular organisms, this shift in metabolism toward anabolic processes is dictated by nutrient availability (Vander Heiden et al. 2009). However, for mammalian cells nutrient uptake is controlled by the signaling pathways that also regulate cell growth and proliferation (Deberardinis et al. 2008; Vander Heiden et al. 2009). These signaling pathways contribute to the development of human cancer, and their inappropriate activation in cancer is accompanied by an increase in nutrient uptake that has long been described as a characteristic feature of malignant cells (Locasale et al. 2009; Levine and Puzio-Kuter 2010).

Additional metabolic changes, such as the increased conversion of glucose to lactate, have also been described in cancer. The increased fermentation of glucose to lactate is referred to as aerobic glycolysis, or the Warburg effect, and is a characteristic feature of many cancer cells and normal proliferating cells (Lunt and Vander Heiden 2011). Aerobic glycolysis is less efficient than the complete oxidative metabolism of glucose in terms of ATP production, and this focus on inefficient ATP production has confounded efforts to understand what advantage aerobic glycolysis may provide for cell proliferation. One hypothesis is that aerobic glycolysis represents a way to generate critical precursors for anabolic processes while 

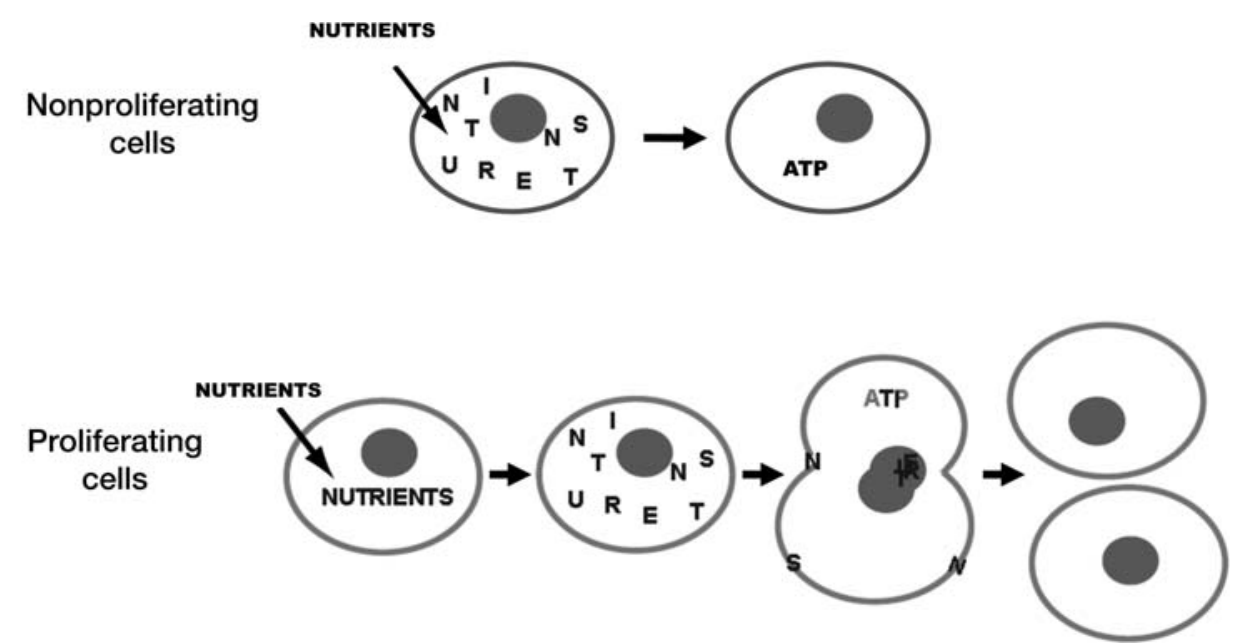

Figure 1. Cell proliferation requires additional nutrient uptake to support duplication of biomass. The otherwise unfavorable reactions required by all cells to maintain homeostasis are coupled to ATP hydrolysis. This requires the constant regeneration of ATP, and as a result all cells rely on nutrients to fuel ATP production. In addition to supporting ATP production, proliferating cells must take up additional nutrients and use those nutrients to generate precursors needed to duplicate the mass of the cell.

retaining adequate ATP production to maintain cell homeostasis (Vander Heiden et al. 2009). It may also allow for adequate redox balance during high rates of glucose uptake. Much is known about how cells regulate metabolism to generate ATP, as well as how specific metabolic building blocks are generated from available nutrients. However, it is poorly understood how metabolism is regulated to produce enough ATP while simultaneously generating all the building blocks in the correct ratios to build a new cell. Here, we review recent work illustrating how metabolic pathways are regulated in proliferating cells to generate some of the key molecules needed to support growth. We also discuss how both genetic and environmental factors can influence the regulation of these pathways to promote cell proliferation in different contexts.

\section{THE REQUIREMENTS OF ANABOLIC METABOLISM}

\section{Cell Proliferation Has Metabolic Needs other than ATP}

Efforts to understand metabolic changes that accompany proliferation have been confounded by attempts to reconcile the relative inefficiency of aerobic glycolysis for ATP production. When coupled to mitochondrial oxidative phosphorylation, the complete catabolism of glucose to $\mathrm{CO}_{2}$ can produce large amounts of ATP. However, other metabolic outputs are also required to support cell growth. For instance, synthesizing a major structural component of cell membranes, a 16-carbon fatty acid (palmitate), from glucose requires ATP as well as carbon precursors and reducing equivalents in the form of nicotinamide adenine dinucleotide phosphate, reduced (NADPH) (Fig. 2). All anabolic processes require a source of carbon and/or nitrogen and many also need reducing power in the form of NADPH (Fig. 3). NADPH, like ATP, must be constantly regenerated to support these biosynthetic processes. Regeneration of NADPH is also required to keep glutathione in the reduced state and maintain a reduced intracellular environment (Lehninger et al. 1993).

Studies of metabolic regulation have largely focused on ATP production, and there is limited understanding of how cells meet these other requirements of cell proliferation (Lunt and Vander Heiden 2011). For example, the pentose phosphate pathway is thought to be the major

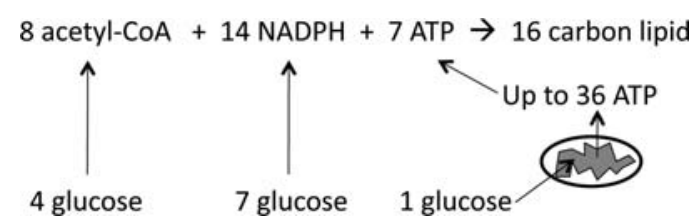

Figure 2. Anabolic metabolism has requirements in addition to ATP. Lipid synthesis to support new membrane production is needed to support the growth and proliferation of many cells. The requirements in terms of carbon units (acetyl-coenzyme $\mathrm{A}, \mathrm{coA}$ ), reducing equivalents (NADPH), and ATP are shown to make a 16-carbon palmitate molecule. Palmitate represents a major structural component of cell membranes. The nutrient "cost" for making each precursor metabolite is shown as a number of glucose equivalents. Even if glycolysis alone is used to generate ATP, fewer than four glucose molecules are needed to make enough ATP for production of a palmitate molecule. The NADPH calculation assumes that two NADPH molecules are generated per glucose via the pentose phosphate pathway. The same glucose molecule can be used to make NADPH and ATP while also generating acetyl-coA, and other pathways and metabolites, such as glutamine, can be used to support lipid synthesis. Nevertheless, the example illustrates that the biosynthesis of many cellular building blocks requires nutrients in excess of those needed for ATP production alone. 


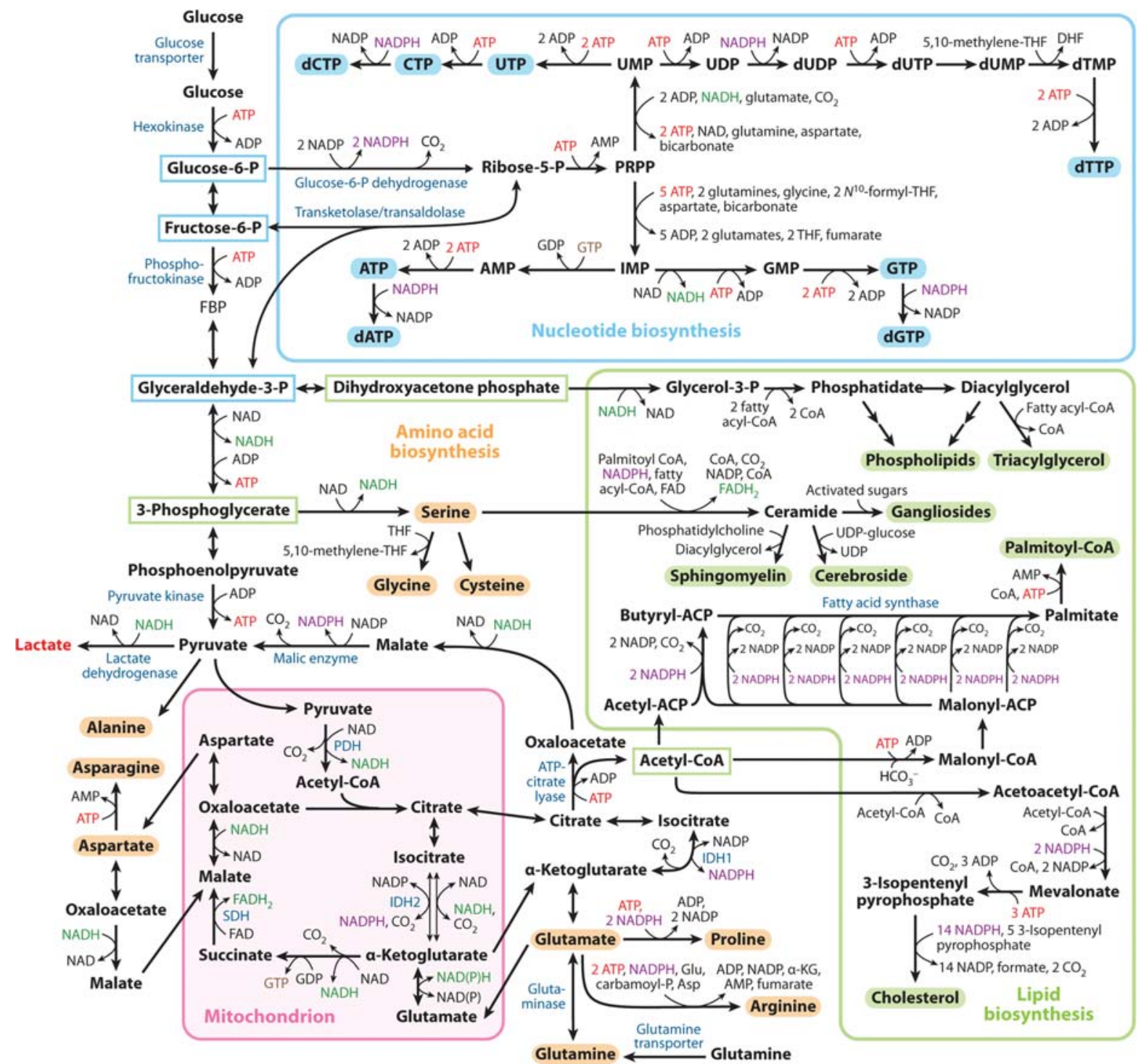

Figure 3. Metabolic pathways active in proliferating cells. This schematic represents our current understanding of how different pathways in central carbon metabolism contribute to biomass precursors. Enzymes that control critical steps and are often overexpressed or mutated in cancer cells are shown in blue. Abbreviations (other than standard nucleotide or amino acid abbreviations): PDH, pyruvate dehydrogenase; SDH, succinate dehydrogenase; IDH, isocitrate dehydrogenase; P, phosphate; PRPP, phosphoribosyl pyrophosphate; $\mathrm{CoA}$, coenzyme A; ACP, acyl carrier protein; THF, tetrahydrofolate; DHF, dihydrofolate; $\alpha \mathrm{KG}, \alpha$-ketoglutarate. (Adapted, with permission, from Lunt and Vander Heiden 2011.)

source of NADPH production in cells. Whereas this is true for some cells, there is increasing evidence that flux through the oxidative pentose phosphate pathway is low in some proliferating cells (Boros et al. 1998, 2000). This suggests other pathways must be used in some situations to provide NAPDH for anabolic reactions. How differential pathway utilization for NADPH generation is regulated in different contexts is not known.

Some work has been done to determine the ATP requirements of both proliferating cancer cells and nonproliferating cells. Indeed, proliferative metabolism does require additional ATP beyond that needed to maintain homeostasis of quiescent cells. However, the incremental increase in ATP demand necessary to support prolifera- tion is small relative to the large ATP consumption needed by all cells to support metabolic processes in the absence of proliferation (Fig. 4) (Kilburn et al. 1969; Locasale and Cantley 2010; Lunt and Vander Heiden 2011). Therefore, we hypothesize that proliferative cell metabolism is adapted to supply carbon, nitrogen, and reducing equivalents to support production of macromolecules needed to build a new cell.

\section{The Growth of Most Proliferating Cells Is Not Limited by ATP}

Most proliferating mammalian cells are exposed to a relatively constant supply of nutrients. There is no evi- 


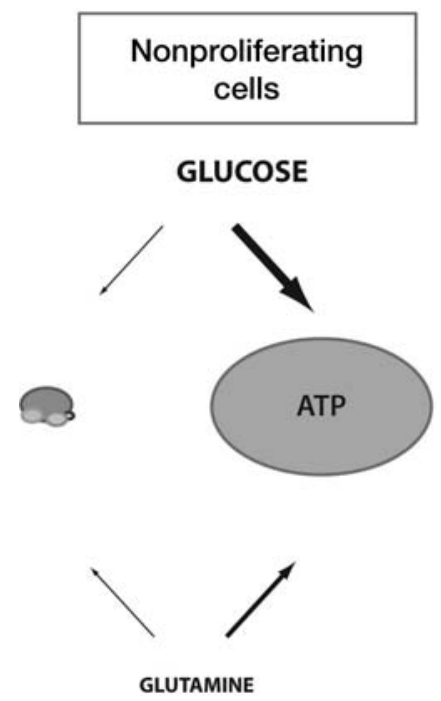

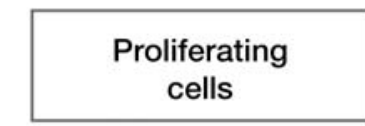

\section{GLUCOSE}

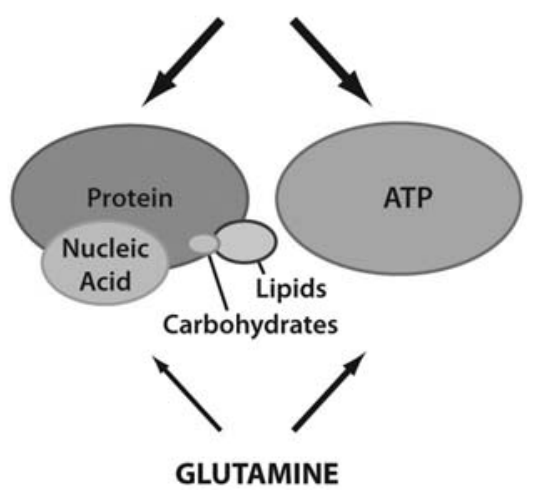

Figure 4. Proliferative cell metabolism involves increased use of nutrients for biosynthesis. Glucose and glutamine are used to support cell growth, and mammalian cells are exposed to a relatively constant supply of these nutrients. Metabolism of other nutrients, including lipids and amino acids, can also be a source of ATP and macromolecular precursors for some cells. The relative ATP requirement is shown to scale based on the calculations presented in $\mathrm{Zu}$ and Guppy (2004). The increase ATP requirement to support proliferation is small relative to the amount of ATP needed to maintain homeostasis in the absence of cell proliferation, arguing that much of the increased nutrient uptake in proliferating cancer cells is used to support biosynthetic reactions. (Adapted, with permission, from Vander Heiden 2011.)

dence that ATP is rate limiting for cell growth under these conditions (Kilburn et al. 1969; Vander Heiden et al. 2009). In fact, sufficient ATP consumption is needed to prevent inhibition of key rate-limiting steps in glycolysis. The phosphofructokinase step of glycolysis in particular is sensitive to the ATP/AMP ratio, and this enzyme controls the entry of glucose metabolites into the downstream steps of glycolysis (Lehninger et al. 1993). Glucose utilization by tumors cells has been shown to be limited by ATP consumption (Scholnick et al. 1973), and a recent study confirmed that for many cancer cells ATP consumption is needed to increase glucose metabolism (Fang et al. 2010). Nevertheless, it has also been argued that increased glycolysis in cancer may allow ATP generation at a faster rate (Koppenol et al. 2011). Indeed, despite a lower yield of ATP per molecule of glucose, when the glucose supply is not limiting, glycolysis alone can produce ATP faster than mitochondrial oxidative phosphorylation (Pfeiffer et al. 2001; Koppenol et al. 2011). However, oxidative phosphorylation supplies the majority of ATP for most proliferating cells: A comparison of data from several cell lines found that glycolysis contributes only about $20 \%$ of the cellular ATP, with the rest provided by the mitochondria ( $\mathrm{Zu}$ and Guppy 2004).

\section{The Organization and Regulation of Metabolic Pathways in Proliferating Cells Is Poorly Understood}

The basic network of reactions that cells used to generate ATP and to synthesize specific macromolecules has been defined for decades (Fig. 3), and the feedback mechanisms ensuring enough ATP is made in different physiological situations are well described (Lehninger et al. 1993). Historically, this knowledge was derived mostly from studies of nonproliferating cells. Until recently, few efforts have been made to understand the metabolic network of proliferating cells. Cancer is a disease of uncontrolled proliferation and therefore provides a model system to understand how pathways are regulated to support growth. Studies from several groups have found that enzymes in central carbon metabolism are regulated by the signal transduction pathways mutated in cancer that control cell proliferation (Deberardinis et al. 2008; Vander Heiden et al. 2009; Cairns et al. 2011). Some of these signaling pathways are also responsive to environmental conditions, including oxygen levels and nutrient availability (Kaelin and Ratcliffe 2008; Zoncu et al. 2011). This suggests that cells integrate growth signals with environmental conditions to actively regulate a metabolic program conducive to cell growth and proliferation.

Understanding the regulation of pyruvate kinase by growth signals has provided insight into how proximal glucose metabolism is controlled in proliferating cells to redirect carbon away from ATP production and into biosynthetic pathways. Additional studies tracking the fate of carbon derived from extracellular nutrients in different genetic and environmental contexts have also illuminated metabolic pathway organization in proliferating cells. These studies are providing new insight into the metabolic programs that different cancer cells use to support proliferation. 

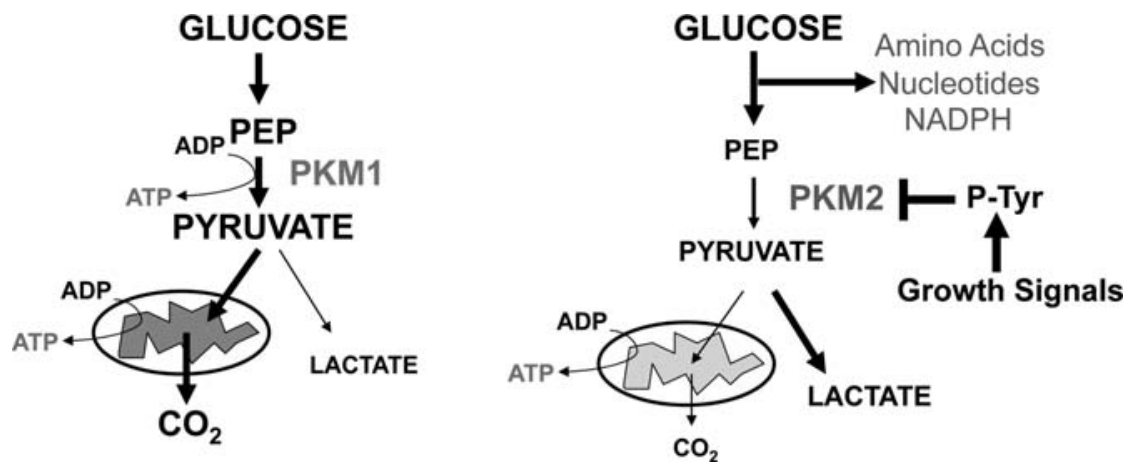

Figure 5. The less active PKM2 isoform of pyruvate kinase is associated with increased lactate production and anabolic metabolism. A schematic representation of how the PKM1 and PKM2 isoform of pyruvate kinase influences glucose metabolism is shown. The PKM1 isoform with high constitutive activity promotes oxidative metabolism and efficient production of ATP. The PKM2 isoform can interact with proteins phosphorylated on tyrosine residues downstream from cell growth signals, and this interaction decreases enzyme activity. Inhibition of PKM2 activity by cell growth signals is associated with increased lactate production and enhanced anabolic metabolism.

\section{PYRUVATE KINASE IS A REGULATED STEP IN PROLIFERATING CELL METABOLISM}

\section{The Less Active PKM2 Isoform Is Expressed in Proliferating Cells}

Pyruvate kinase catalyzes the conversion of phosphoenolpyruvate (PEP) and ADP to ATP and pyruvate and is a key regulated step in the latter half of glycolysis (Figs. 4 and 5) (Lehninger et al. 1993). Four isoforms of pyruvate kinase are present in mammals, and the M2 isoform (PKM2) is the isoform expressed in both cancer cells and normal proliferating cells (Mazurek 2010). PKM2 is a product of the $P K-M$ gene, which is alternatively spliced by differential selection of a single exon to produce either the PKM1 or PKM2 isoform (Takenaka et al. 1996). Consistent with the expression of PKM2 in proliferating cells, this isoform promotes both aerobic glycolysis and anabolic metabolism (Christofk et al. 2008a). Many nonproliferating differentiated tissues that rely on oxidative metabolism express PKM1. Forcing tumor cells to express the more enzymatically active PKM1 isoform leads to increased oxidative metabolism and the inability to form xenograft tumors in mice (Christofk et al. 2008a). This suggests that PKM2 plays a critical role in proliferative metabolism in vivo. The differentially spliced exons that distinguish PKM1 from PKM2 are identical in size and encode a 56-amino-acid stretch of the enzyme that allows PKM2, but not PKM1, to be allosterically activated by the upstream glycolytic intermediate fructose-1,6-bisphosphate (FBP) (Dombrauckas et al. 2005). The unique region that distinguishes PKM2 from the other pyruvate kinase isoforms also allows for inhibition of enzymatic activity via binding to proteins phosphorylated on tyrosine downstream from cell growth signals (Christofk et al. 2008b). Interestingly, decreased PKM2 activity caused by the interaction with phosphotyrosine-containing proteins leads to decreased glucose incorporation into lipids (Fig. 6A) and impaired cell proliferation (Christofk et al. 2008b). This suggests that an important event downstream from growth factor signaling is a decrease in PKM2 activity and that this decrease in activity contributes to efficient incorporation of nutrients into biomass (Fig. 5).

\section{Cells Can Proliferate with Little or no Pyruvate Kinase Activity}

Glucose metabolism by aerobic glycolysis to produce lactate is characteristic of many cancer cells. Lactate is produced directly from pyruvate, and pyruvate is one of the products of pyruvate kinase. Therefore, it is surprising that decreased pyruvate kinase activity in cancer cells is associated with increased lactate production (Christofk et al. 2008a). Pyruvate can also be metabolized to compounds required for cell growth (Fig. 3). Acetyl-coA is a major carbon precursor used for lipid synthesis and can be derived directly from pyruvate. However, as discussed above, increased lipid synthesis is also associated with decreased pyruvate kinase activity in PKM2-expressing cells (Christofk et al. 2008a,b; Fig. 6A). Nevertheless, PKM2-expressing cells have more pyruvate than cells engineered to express PKM1 (Christofk et al. 2008a), and it remains unclear how lower pyruvate kinase activity promotes metabolism downstream of pyruvate.

Proliferating cells can convert PEP to pyruvate in the absence of pyruvate kinase. When pyruvate kinase expression is decreased using shRNA, cells retain the ability to proliferate despite retaining very little pyruvate kinase activity (Christofk et al. 2008a; Vander Heiden et al. 2010; Fig. 6B). Glucose remains the major carbon source used by these cells (not shown), and many anabolic reactions require metabolism of glucose to metabolites downstream from PEP (Fig. 3). These findings argue that some proliferating cells rely on pyruvate kinase-independent glucose metabolism.

\section{Proliferating Cells Utilize Another Enzyme to Convert PEP to Pyruvate that Influences Regulation of Glucose Metabolism}

There is biochemical evidence that an activity other than pyruvate kinase can convert PEP to pyruvate (Vander 
A

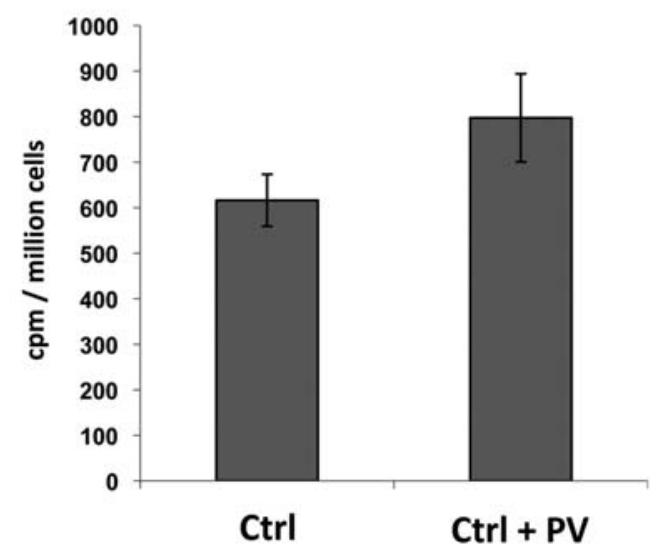

B

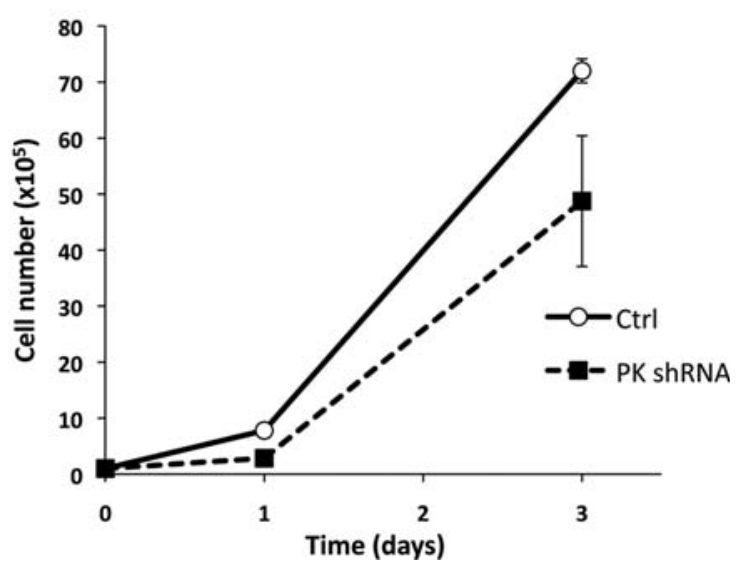

Figure 6. Anabolic metabolism and cell growth occur with low pyruvate kinase activity. $(A)$ Increased phosphotyrosine signaling decreases pyruvate kinase activity and promotes incorporation of glucose carbon into lipids (Christofk et al. 2008b). H1299 lung cancer cells were grown in $\left[6-{ }^{14} \mathrm{C}\right]$-glucose, and incorporation of ${ }^{14} \mathrm{C}$-labeled carbon into the extracted lipid fraction determined by scintillation counting in the presence or absence of pervandate $(\mathrm{PV})$ to increase phosphotyrosine signaling is shown (for experimental details, see Christofk et al. 2008b). cpm, counts per minute. (B) Cells continue to proliferate with very little pyruvate kinase. Doxycycline-inducible shRNA constructs targeting a PKM message were introduced into A13 pancreatic cancer cells expressing a reverse tetracycline transactivator. Cell proliferation was assessed by cell counting over time in the absence (ctrl) or presence (PK shRNA) of doxycycline to knock down pyruvate kinase expression as shown. Similar to data reported in Christofk et al. (2008a) with H1299 cells, these cells continue to grow and divide despite having very little pyruvate kinase.

Heiden et al. 2010). This activity is associated with transfer of the phosphate group from PEP to the catalytic histidine residue of phosphoglycerate mutase (PGAM) (Fig. 7) (Vander Heiden et al. 2010). Phosphorylation of PGAM primes the enzyme for catalytic activity, creating a positive feedback loop where PEP increases PGAM activity. The rate of glucose metabolism exceeds the stoichiometry of PGAM to accept the phosphate from PEP; thus a mechanism to release a free phosphate group is needed to allow bulk PEP to pyruvate conversion in the absence of pyruvate
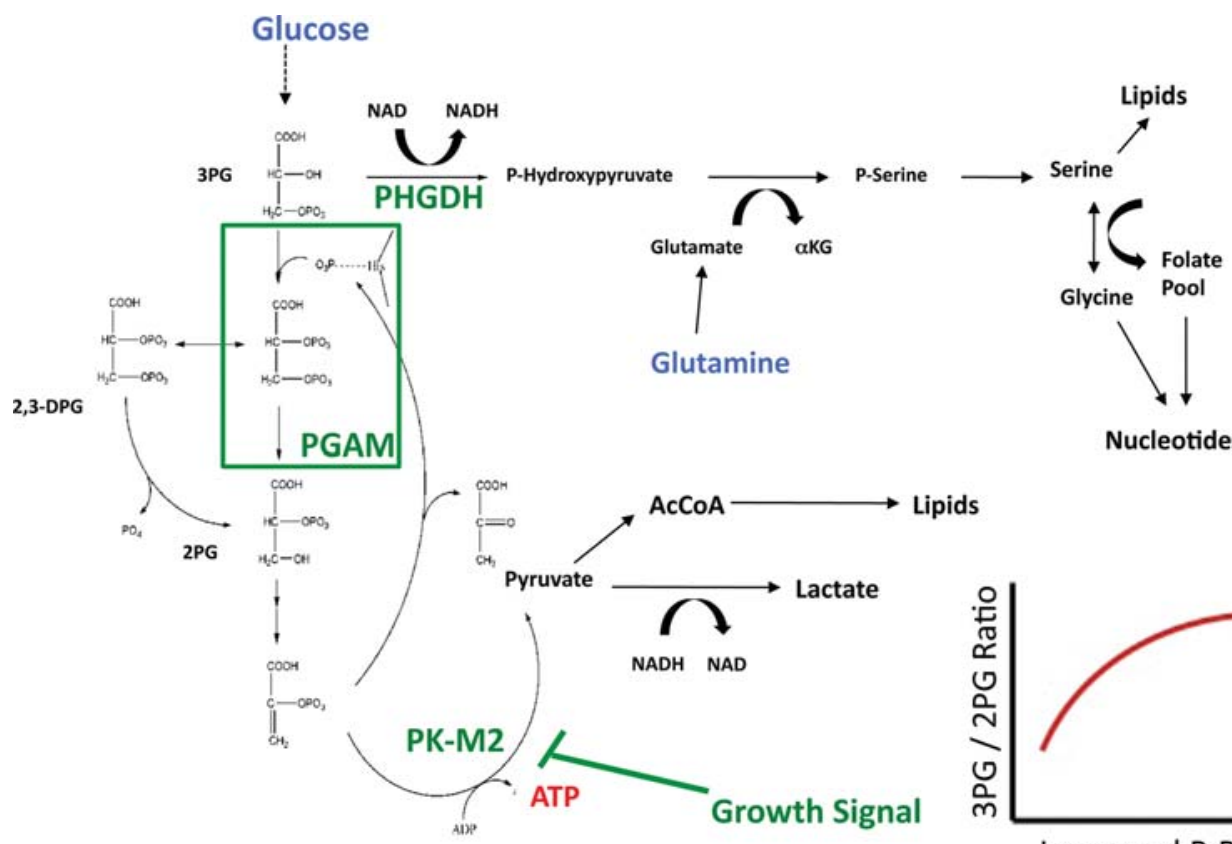

Nucleotides

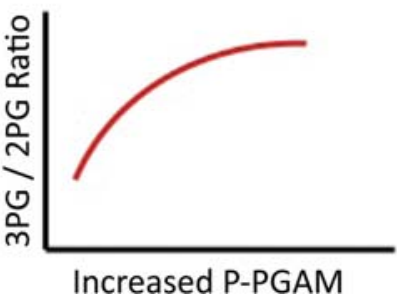

Figure 7. Pyruvate kinase-independent glycolysis redirects glycolytic carbon into the serine and glycine biosynthesis pathway. A schematic representation of how the lower part of glycolysis is regulated in PKM2-expressing cells to promote serine biosynthesis is shown. When PKM2 activity is inhibited by phosphotyrosine-mediated growth signals, phosphoenolpyruvate (PEP) to pyruvate conversion can still occur via a partially characterized activity. This activity is associated with a feedback loop where the phosphate from PEP can be transferred to the catalytic histidine of PGAM. Increased phospho-PGAM (P-PGAM) increases the activity of this enzyme and is predicted to increase the ratio of 3-phosphoglycerate (3PG) to 2-phosphoglycerate (2PG) and redirect glycolytic carbon into the serine and glycine biosynthesis pathway. Metabolites in this pathway are important precursors for biosynthetic reactions, and serine pathway flux is also seen in cancer cells with increased expression of phosphoglycerate dehydrogenase (PHGDH) resulting from gene-copy-number gain. 
kinase. Cells have a phosphatase that converts the 2,3diphosphoglycerate intermediate generated by the PGAM mutase reaction to 2-phosphoglycerate (Cho et al. 2008). It is also possible that the PEP-dependent PGAM phosphorylating activity releases phosphate from PEP to generate pyruvate via another mechanism. Nevertheless, the presence of an alternative glycolytic pathway provides an explanation for how cells grow in the absence of pyruvate kinase, as well as how cells expressing the less active PKM2 isoform can maintain high glucose to lactate flux.

This alternative activity to convert PEP to pyruvate is active in PKM2-expressing cells with low pyruvate kinase activity (Vander Heiden et al. 2010). ATP is not generated in this reaction. Because the net ATP produced by glycolysis is derived from pyruvate kinase, engaging the alternative glycolytic pathway allows glucose assimilation into the metabolic network without making ATP. Therefore, this may be a mechanism to prevent accumulation of a high ATP/AMP ratio that would otherwise inhibit glucose metabolism and allow efficient biomass accumulation.

\section{REDIRECTION OF GLUCOSE CARBON INTO SERINE BIOSYNTHESIS TO SUPPORT CELL PROLIFERATION}

\section{Redirection of Glycolytic Metabolites into the Serine Biosynthesis Pathway Is a Consequence of PKM2 Regulation}

PKM2 inhibition to engage an alternative glycolytic pathway has additional implications for metabolic regulation. This alternative pathway is associated with a positive feedback loop that increases PGAM activity such that PEP promotes the production of PEP (Fig. 7). One consequence of this feedback regulation is an increase in the 3phosphoglycerate (3-PG) to 2-phosphoglycerate ratio that accompanies increasing PGAM activation (J Locasale, unpubl.). Because the feedback loop effectively increases the relative concentration of 3-PG, this increase in chemical potential favors the flow of glucose metabolites into other pathways that branch from glycolysis at 3-PG. Once such pathway is used to generate serine and glycine from glucose (de Koning et al. 2003). Therefore, use of the alternative glycolytic pathway promoted by PKM2 inhibition is predicted to redirect glucose metabolism into serine biosynthesis (Fig. 7). In support of this prediction, many cancer cells exhibit increased flux from glucose into serine and glycine biosynthesis (Locasale et al. 2011). Serine and glycine are important precursors to make proteins, lipids, and nucleic acids (Figs. 3 and 7). Hence, this is one mechanism by which decreased pyruvate kinase activity can promote anabolic metabolism.

\section{Gene Amplification to Increase Expression of PHGDH Can Also Promote Increased Dependence on the Serine Biosynthesis Pathway in Some Cancer Cells}

Additional evidence supports the importance of redirecting glucose carbon into the serine biosynthesis path- way for cell proliferation. The PHGDH gene encodes phosphoglycerate dehydrogenase, the first enzyme in the serine biosynthesis pathway branching from glycolysis (Fig. 7), and $P H G D H$ is amplified in a subset of human cancers (Locasale et al. 2011). PHGDH copy-number gain causes increased $\mathrm{PHGDH}$ protein expression and is observed most frequently in melanoma and breast cancer. Increased PGHDH expression results in increased flux of glucose carbon into serine biosynthesis, and proliferation of cells with $P H G D H$ copy-number gain is blocked by shRNA-mediated knockdown of $P H G D H$ expression (Locasale et al. 2011; Possemato et al. 2011). Thus, for human cancer cells, at least two different mechanisms exist to increase the flow of glucose carbon into serine biosynthesis: phosphotyrosine growth signal-mediated regulation of PKM2 and $P H G D H$ copy-number gain. Whether increases in PHGDH by mechanisms other than $\mathrm{PHGDH}$ amplification are used to promote cell proliferation under some physiological conditions remains to be determined.

\section{GLUTAMINE CARBON CAN BE USED AS A PRECURSOR FOR LIPID SYNTHESIS}

\section{Reductive Metabolism of Glutamine to Produce Cytosolic Acetyl-coA}

Glycolysis and the serine biosynthesis pathway supply important molecules for anabolic metabolism; however, important biosynthetic precursors are also derived from intermediates in the tricarboxylic acid (TCA) cycle (Fig. 3). Pyruvate can be converted to acetyl-coA in the mitochondria. This is the first step in the complete oxidation of glucose in the TCA cycle that allows maximal ATP production from glucose when coupled to oxidative phosphorylation. However, acetyl-coA can also be exported from the mitochondria via the citrate shuttle for use as a two-carbon building block in anabolic reactions in the cytosol. For instance, acetyl-coA is a source of carbon for lipid synthesis. Many proliferating cells synthesize lipids de novo (Ookhtens et al. 1984; Menendez and Lupu 2007), and glucose is a major source of acetylcoA in cells (Hatzivassiliou et al. 2005; Wellen et al. 2009). However, glutamine can also be a source of carbon for acetyl-coA (Yoo et al. 2004, 2008; DeBerardinis et al. 2007), and it may be the principle source of this important biosynthetic precursor under some physiological conditions (Metallo et al. 2011).

Proliferating mammalian cells use glutamine as a source of nitrogen and as a key anapleurotic carbon source to replenish metabolites depleted from the TCA cycle for biosynthesis (Fig. 3) (DeBerardinis et al. 2007; Dang 2010). Glutamine can provide carbon for lipid synthesis by oxidative metabolism through the TCA cycle to produce malate, which when converted to pyruvate can be decarboxylated to yield acetyl-coA in the mitochondria followed by export to the cytosol via the citrate shuttle. This route of glutamine metabolism, termed glutaminolysis, can fuel mitochondrial ATP generation, serve as a source of NADPH by the action of malic 


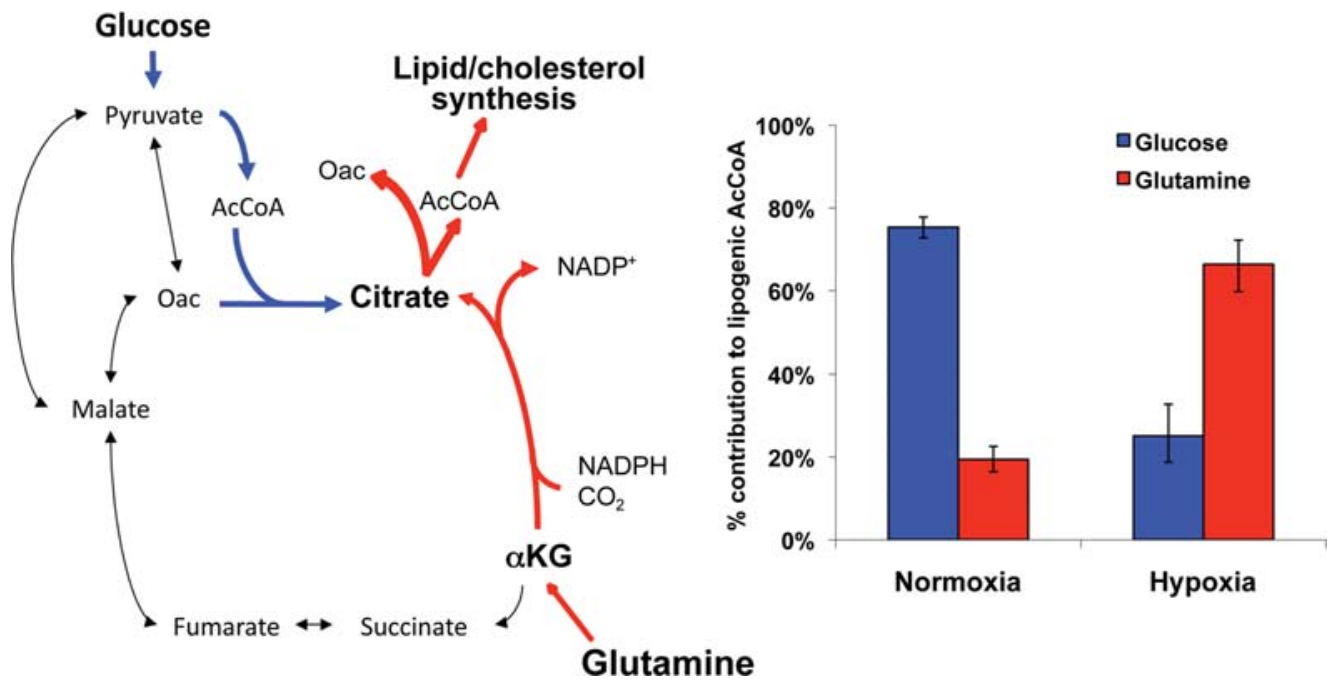

Figure 8. The source of carbon used to generate lipogenic acetyl-coA switches from glucose to glutamine when oxygen levels are low. Glucose is used to generate acetyl-coA (AcCoA) in cells under standard tissue culture conditions (glucose oxidation; pathway shown in blue). However, when oxygen is limiting, as is the case for many cells in vivo, reductive metabolism of glutamine is used to synthesize AcCoA used for lipid synthesis (glutamine reduction; red pathway). Data shown for percentage of contribution to lipogenic AcCoA were obtained using ${ }^{13} \mathrm{C}$-labeled glucose or ${ }^{13} \mathrm{C}$-labeled glutamine from proliferating HCT116 colon cancer cells grown under standard tissue culture conditions (normoxia) or 1\% oxygen (hypoxia). Detailed methods are described in Metallo et al. (2011). Oac, oxaloacetate; $\alpha \mathrm{KG}, \alpha$-ketoglutarate.

enzyme, and supply anapleurotic carbon to the TCA cycle (DeBerardinis et al. 2007; Dang 2010). However, glutamine can also be used to generate cytosolic acetyl-coA via a reductive pathway involving direct conversion of $\alpha$-ketoglutarate and $\mathrm{CO}_{2}$ to citrate (Yoo et al. 2008; Metallo et al. 2011). This citrate can then be used as a source of acetyl-coA in the cytosol (Fig. 8).

\section{Low Oxygen Levels or VHL Loss Promoted Reductive Glutamine Metabolism to Support Lipid Synthesis}

Whereas the reductive metabolism of glutamine was known to occur in some differentiated tissues (Yoo et al. 2008), recent evidence using isotope-labeled carbon tracing suggests that the reductive metabolism of glutamine can be a major pathway for lipid production when cells are hypoxic (Metallo et al. 2011). Reductive glutamine metabolism is a minor source of lipogenic acetyl-coA when oxygen levels are high, but glutamine becomes the major source of carbon for lipid synthesis when cells are proliferating under low-oxygen conditions (Fig. 8). This switch from glucose to glutamine as a source of carbon for lipogenesis is mediated in part by the VHL/ HIF (von Hippel-Landau gene product/hypoxia-inducible factor) signaling pathway that senses oxygen levels in cells (Kaelin 2008). Low-oxygen levels lead to HIF stabilization and increased expression of pyruvate dehydrogenase kinase 1 (Kim et al. 2006; Papandreou et al. 2006). Pyruvate dehydrogenase kinase is a negative regulator of pyruvate dehydrogenase, the enzyme that converts pyruvate to acetyl-coA. Less acetyl-coA production from pyruvate results in less citrate production, and the decreased citrate levels observed in hypoxic cells provide one driving force for reductive metabolism of $\alpha$-ketoglutarate to citrate (Metallo et al. 2011). The VHL tumor suppressor protein is a ubiquitin E3 ligase that degrades HIF when oxygen levels are high and is frequently lost in some human cancers (Kaelin 2008). Consistent with this pathway influencing the use of glutamine carbon for lipid synthesis, VHL-deficient renal cell cancer lines use reductive glutamine metabolism for lipogenesis even when oxygen levels are high (Metallo et al. 2011). In hypoxic microenvironments in vivo, glucose is also likely to be limited. Because glucose carbon is necessary for nucleic acid biosynthesis (Metallo and Vander Heiden 2010; Wellen et al. 2010), use of reductive glutamine metabolism for lipid synthesis may allow cells to more efficiently distribute available nutrients under conditions of metabolic stress. These findings illustrate another example of how metabolic pathway organization is altered to provide biosynthetic precursors that support cell proliferation.

\section{CONCLUSIONS}

Cellular metabolism is adapted to support physiological processes in cells. These include housekeeping functions as well as functions unique to specific cell types. Evidence is accumulating that metabolism is actively regulated to facilitate a metabolic program that allows cells to adapt to physiological situations. For proliferating cells, this involves a reorganization of metabolic pathways to generate precursors needed to create biomass. Genetic factors such as pyruvate kinase isoform selection, PHGDH expression, and VHL expression can influence the flow of carbon through the metabolic network. The cell environment can also impact which pathways 
are used to support proliferation, potentially as a means to efficiently distribute available nutrients into pathways that generate biomass. Thus, proliferating cells in different genetic and environmental contexts can adapt metabolism to support a biosynthetic program that is matched to specific cellular conditions. Our knowledge of how cells actively regulate metabolism to achieve these goals is incomplete. However, understanding the metabolic solutions different cells use to support proliferation will be critical to understanding metabolic changes associated with various disease states such as cancer.

\section{ACKNOWLEDGMENTS}

We thank B. Bevis for helpful comments on the manuscript. M.V.H. acknowledges support from the Burroughs Wellcome Fund, the Damon Runyon Cancer Research Foundation, the Smith Family, the Starr Cancer Consortium, and the National Institutes of Health.

\section{REFERENCES}

Boros LG, Lee PW, Brandes JL, Cascante M, Muscarella P, Schirmer WJ, Melvin WS, Ellison EC. 1998. Nonoxidative pentose phosphate pathways and their direct role in ribose synthesis in tumors: Is cancer a disease of cellular glucose metabolism? Med Hypotheses 50: 55-59.

Boros LG, Torday JS, Lim S, Bassilian S, Cascante M, Lee WN. 2000. Transforming growth factor $\beta 2$ promotes glucose carbon incorporation into nucleic acid ribose through the nonoxidative pentose cycle in lung epithelial carcinoma cells. Cancer Res 60: 1183-1185.

Cairns RA, Harris IS, Mak TW. 2011. Regulation of cancer cell metabolism. Nat Rev Cancer 11: 85-95.

Cho J, King JS, Qian X, Harwood AJ, Shears SB. 2008. Dephosphorylation of 2,3-bisphosphoglycerate by MIPP expands the regulatory capacity of the Rapoport-Luebering glycolytic shunt. Proc Natl Acad Sci 105: 5998-6003.

Christofk HR, Vander Heiden MG, Harris MH, Ramanathan A, Gerszten RE, Wei R, Fleming MD, Schreiber SL, Cantley LC. 2008a. The M2 splice isoform of pyruvate kinase is important for cancer metabolism and tumour growth. Nature 452: 230 233.

Christofk HR, Vander Heiden MG, Wu N, Asara JM, Cantley LC. 2008b. Pyruvate kinase M2 is a phosphotyrosine-binding protein. Nature 452: 181-186.

Dang CV. 2010. Glutaminolysis: Supplying carbon or nitrogen or both for cancer cells? Cell Cycle 9: 3884-3886.

DeBerardinis RJ, Mancuso A, Daikhin E, Nissim I, Yudkoff M, Wehrli S, Thompson CB. 2007. Beyond aerobic glycolysis: Transformed cells can engage in glutamine metabolism that exceeds the requirement for protein and nucleotide synthesis. Proc Natl Acad Sci 104: 19345-19350.

DeBerardinis RJ, Lum JJ, Hatzivassiliou G, Thompson CB. 2008. The biology of cancer: Metabolic reprogramming fuels cell growth and proliferation. Cell Metab 7: 11-20.

de Koning TJ, Snell K, Duran M, Berger R, Poll-The BT, Surtees R. 2003. L-Serine in disease and development. Biochem J 371: $653-661$.

Dombrauckas JD, Santarsiero BD, Mesecar AD. 2005. Structural basis for tumor pyruvate kinase M2 allosteric regulation and catalysis. Biochemistry 44: 9417-9429.

Fang M, Shen Z, Huang S, Zhao L, Chen S, Mak TW, Wang X. 2010. The ER UDPase ENTPD5 promotes protein $N$-glycosylation, the Warburg effect, and proliferation in the PTEN pathway. Cell 143: $711-724$.

Hatzivassiliou G, Zhao F, Bauer DE, Andreadis C, Shaw AN, Dhanak D, Hingorani SR, Tuveson DA, Thompson CB. 2005.
ATP citrate lyase inhibition can suppress tumor cell growth. Cancer Cell 8: 311-321.

Kaelin WG Jr. 2008. The von Hippel-Lindau tumour suppressor protein: $\mathrm{O}_{2}$ sensing and cancer. Nat Rev Cancer 8: 865-873.

Kaelin WG Jr, Ratcliffe PJ. 2008. Oxygen sensing by metazoans: The central role of the HIF hydroxylase pathway. Mol Cell 30: 393-402.

Kilburn DG, Lilly MD, Webb FC. 1969. The energetics of mammalian cell growth. J Cell Sci 4: 645-654.

Kim JW, Tchernyshyov I, Semenza GL, Dang CV. 2006. HIF-1mediated expression of pyruvate dehydrogenase kinase: A metabolic switch required for cellular adaptation to hypoxia. Cell Metab 3: 177-185.

Koppenol WH, Bounds PL, Dang CV. 2011. Otto Warburg's contributions to current concepts of cancer metabolism. Nat Rev Cancer 11: 325-337.

Lehninger AL, Nelson DL, Cox MM. 1993. Principles of biochemistry. Worth, New York.

Levine AJ, Puzio-Kuter AM. 2010. The control of the metabolic switch in cancers by oncogenes and tumor suppressor genes. Science 330: 1340-1344.

Locasale JW, Cantley LC. 2010. Altered metabolism in cancer. BMC Biol 8: 88 .

Locasale JW, Cantley LC, Vander Heiden MG. 2009. Cancer's insatiable appetite. Nat Biotechnol 27: 916-917.

Locasale JW, Grassian AR, Melman T, Lyssiotis CA, Mattaini KR, Bass AJ, Heffron G, Metallo CM, Muranen T, Sharfi H, et al. 2011. Phosphoglycerate dehydrogenase diverts glycolytic flux and contributes to oncogenesis. Nat Genet 43: 869-874.

Lunt SY, Vander Heiden MG. 2011. Aerobic glycolysis: Meeting the metabolic requirements of cell proliferation. Annu Rev Cell Dev Biol 27: 441-464.

Mazurek S. 2010. Pyruvate kinase type M2: A key regulator of the metabolic budget system in tumor cells. Int J Biochem Cell Biol 43: 969-980.

Menendez JA, Lupu R. 2007. Fatty acid synthase and the lipogenic phenotype in cancer pathogenesis. Nat Rev Cancer 7: 763-777.

Metallo CM, Vander Heiden MG. 2010. Metabolism strikes back: Metabolic flux regulates cell signaling. Genes Dev 24: 2717-2722.

Metallo CM, Gameiro PA, Bell EL, Mattaini KR, Yang J, Hiller K, Jewell CM, Johnson ZR, Irvine DJ, Guarente L, et al. 2011. Reductive glutamine metabolism by IDH1 mediates lipogenesis under hypoxia. Nature doi: 10.1038/nature 10602.

Ookhtens M, Kannan R, Lyon I, Baker N. 1984. Liver and adipose tissue contributions to newly formed fatty acids in an ascites tumor. Am J Physiol 247: R146-R153.

Papandreou I, Cairns RA, Fontana L, Lim AL, Denko NC. 2006. HIF-1 mediates adaptation to hypoxia by actively downregulating mitochondrial oxygen consumption. Cell Metab 3: 187-197.

Pfeiffer T, Schuster S, Bonhoeffer S. 2001. Cooperation and competition in the evolution of ATP-producing pathways. Science 292: 504-507.

Possemato R, Marks KM, Shaul YD, Pacold ME, Kim D, Birsoy K, Sethumadhavan S, Woo HK, Jang HG, Jha AK, et al. 2011. Functional genomics reveal that the serine synthesis pathway is essential in breast cancer. Nature 476: 346-350.

Scholnick P, Lang D, Racker E. 1973. Regulatory mechanisms in carbohydrate metabolism. IX. Stimulation of aerobic glycolysis by energy-linked ion transport and inhibition by dextran sulfate. J Biol Chem 248: 5175.

Takenaka M, Yamada K, Lu T, Kang R, Tanaka T, Noguchi T. 1996. Alternative splicing of the pyruvate kinase $M$ gene in a minigene system. Eur J Biochem 235: 366-371.

Vander Heiden MG. 2011. Targeting cancer metabolism: A therapeutic window opens. Nat Rev Drug Discov 10: 671-684.

Vander Heiden MG, Cantley LC, Thompson CB. 2009. Understanding the Warburg effect: The metabolic requirements of cell proliferation. Science 324: 1029-1033. 
Vander Heiden MG, Locasale JW, Swanson KD, Sharfi H, Heffron GJ, Amador-Noguez D, Christofk HR, Wagner G, Rabinowitz JD, Asara JM, et al. 2010. Evidence for an alternative glycolytic pathway in rapidly proliferating cells. Science 329: 1492-1499.

Wellen KE, Hatzivassiliou G, Sachdeva UM, Bui TV, Cross JR, Thompson CB. 2009. ATP-citrate lyase links cellular metabolism to histone acetylation. Science 324: 1076-1080.

Wellen KE, Lu C, Mancuso A, Lemons JM, Ryczko M, Dennis JW, Rabinowitz JD, Coller HA, Thompson CB. 2010. The hexosamine biosynthetic pathway couples growth factorinduced glutamine uptake to glucose metabolism. Genes Dev 24: $2784-2799$.
Yoo H, Stephanopoulos G, Kelleher JK. 2004. Quantifying carbon sources for de novo lipogenesis in wild-type and IRS-1 knockout brown adipocytes. J Lipid Res 45: 13241332.

Yoo H, Antoniewicz MR, Stephanopoulos G, Kelleher JK. 2008. Quantifying reductive carboxylation flux of glutamine to lipid in a brown adipocyte cell line. J Biol Chem 283: $20621-$ 20627.

Zoncu R, Efeyan A, Sabatini DM. 2011. mTOR: From growth signal integration to cancer, diabetes and ageing. Nat Rev Mol Cell Biol 12: 21-35.

Zu XL, Guppy M. 2004. Cancer metabolism: Facts, fantasy, and fiction. Biochem Biophys Res Commun 313: 459-465. 


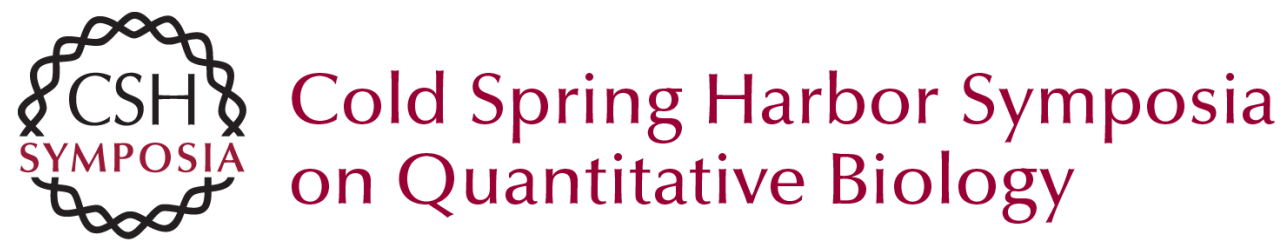

\section{Metabolic Pathway Alterations that Support Cell Proliferation}

M.G. Vander Heiden, S.Y. Lunt, T.L. Dayton, et al.

Cold Spring Harb Symp Quant Biol 2011 76: 325-334 originally published online January 19, 2012

Access the most recent version at doi:10.1101/sqb.2012.76.010900

References This article cites 42 articles, 14 of which can be accessed free at: http://symposium.cshlp.org/content/76/325.full.html\#ref-list-1

\section{License}

Email Alerting Receive free email alerts when new articles cite this article - sign up in Service the box at the top right corner of the article or click here. 